\title{
Spectral Electron Tomography as a Quantitative Technique to Investigate Functional Nanomaterials.
}

\author{
Sara Bals ${ }^{1}$, Daniele Zanaga ${ }^{1}$, Bart Goris ${ }^{1}$, M. Pfannmöller ${ }^{1}$, S. Turner $^{1}$ and Bert Freitag ${ }^{2}$ \\ 1. EMAT, University of Antwerp, Antwerp, Belgium. \\ 2. FEI Company, P.O. Box 80066, KA 5600 Eindhoven, The Netherlands.
}

Electron tomography has become a versatile tool to investigate a broad variety of (hetero)nanostructures in 3 dimensions (3D) [1]. Recent developments even pushed the resolution of the technique to the atomic level [2-4]. In addition to 3D structural characterization, 3D spectral information containing compositional, valency and electronic information can be obtained by 3D X-ray Energy Dispersive Spectroscopy (XEDS) and electron energy loss spectroscopy (EELS).

In an increasing number of studies, XEDS is combined with tomography. These studies rely on newly developed XEDS detectors [5] such as the Super-X detection system, which consists of 4 individual detectors, symmetrically arranged around the TEM sample. Although qualitative results obtained by XEDS tomography have already been reported, [6] it remains difficult to obtain quantitative information by $3 \mathrm{D}$ XEDS and therefore further progress is required. By using the Super-X detector, one is able to largely overcome problems that were previously related to extreme shadowing of XEDS detectors, but some shadowing remains. [7] Since these shadowing effects will change for different tilt angles, the projection principle for electron tomography is no longer fulfilled. In this presentation, we propose a novel and straightforward approach to minimize the impact of shadowing effects during XEDS tomography. The method relies on a synergistic combination of XEDS data, quantified using the $\zeta$-factor approach and 3D high angle annular dark field scanning transmission electron microscopy (HAADFSTEM). HAADF-STEM yields a relatively high signal to noise ratio, and does not suffer from shadowing effects other than those related to the "missing wedge". XEDS, on the other hand, yields chemical information, even when no prior knowledge of the sample is available or for samples that contain elements with a small difference in atomic number Z. As a proof of principle, we will here apply our methodology to different nanostructures containing a mix of $\mathrm{Au}$ and $\mathrm{Ag}$.

In addition to 3D compostional data by XEDS, information on valency can be extracted from EELS measurements. Recently, fine structure changes to the $\mathrm{Ce}_{4,5}$ edge in the core-loss region of the EELS spectrum were used to investigate valence changes at the surface of $\mathrm{CeO}_{2}$ nanoparticles. This is illustrated in Figure 1, where the morphology of a truncated ceria nanoparticle is presented along with spectra that are extracted from the 3D regions indicated in Figure 1a. From Figure 1b, it is obvious that small differences in the spectra can be observed for voxels that are located in the inner part of the reconstruction and voxels that are located at the surface. These differences directly correspond to a $\mathrm{Ce}^{4+}$ $\rightarrow \mathrm{Ce}^{3+}$ surface reduction. [8] These unique experiments revealed a clear facet-dependent reduction shell at the surface of ceria nanoparticles, invisible to modern high resolution TEM structural imaging techniques.

Finally, we will discuss the power of spectral tomography to reconstruct entire volume plasmon spectra of materials in organic photovoltaic tandem cells. [9] As illustrated in Figure 2, such experiments provide 3D structural correlations and compositional mapping for these materials, which is far from straightforward using conventional electron tomography due to a lack of contrast. [10] 


\section{References:}

[1] PA Midgley, RE Dunin-Borkowski Nature Materials 8 (2009) 271

[2] S Van Aert, K.J. Batenburg et al, Nature 470 (2011) 374

[3] MC Scott, C-C Chen et al, Nature 483 (2012) 444

[4] B Goris, S Bals et al, Nature Materials 11 (2012) 930

[5] P. Schlossmacher, D. Klenov, et al, Microscopy today 18 (2010) 14

[6] B. Goris, L. Polavarapu, et al, Nano Letters 14 (2014) 3220

[7] T. J. Slater, A. Janssen, et al, Ultramicroscopy 162 (2016) 61

[8] B Goris, S Turner, et al, ACS Nano 8 (2014) 1087

[9] M. Pfannmoeller, H. Heidari, et al, Nano Letters 15 (2015) 6634

[10] The authors acknowledge financial support from European Research Council (ERC Starting Grant \#335078-COLOURATOMS). The work was supported by the Flemish Fund for Scientific Research

(FWO Vlaanderen) through PhD research grant to B.G. The authors would like to thank Prof. L. Liz-

Marzán for the provision of the samples and fruitful discussions.
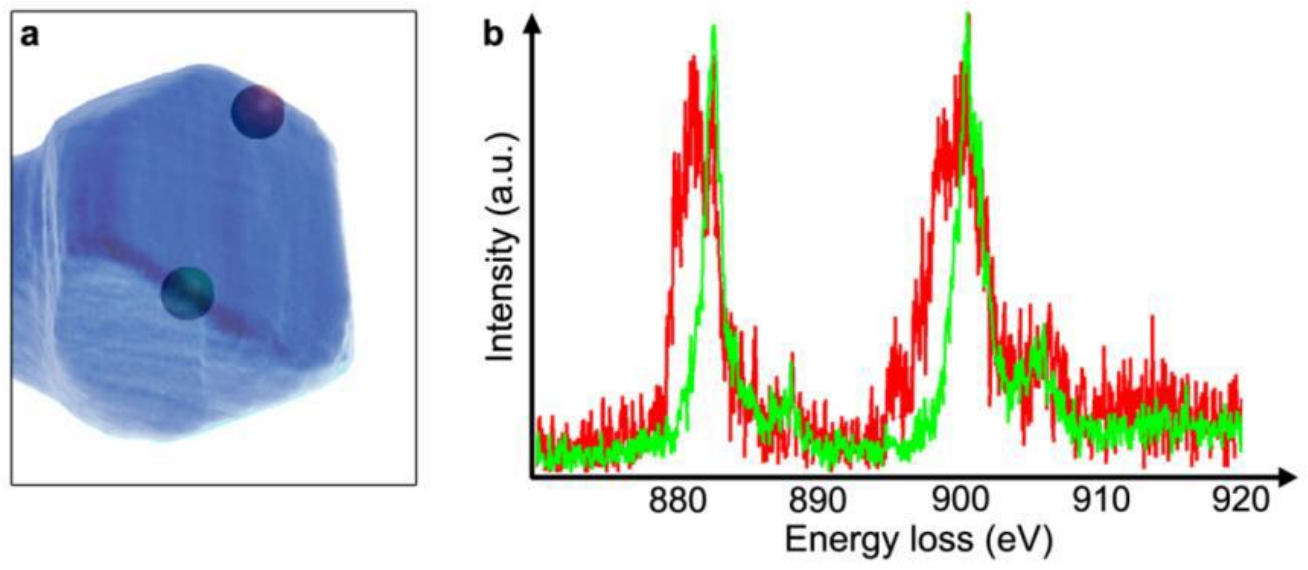

Figure 1. Figure 1: (a) 3D visualisation of the morphology of a reconstructed ceria nanoparticle. At 2 different positions (indicated in a), $3 * 3 * 3$ voxel averaged $\mathrm{Ce}$ $\mathrm{M}_{4,5}$ edge EELS spectra are extracted in (b).
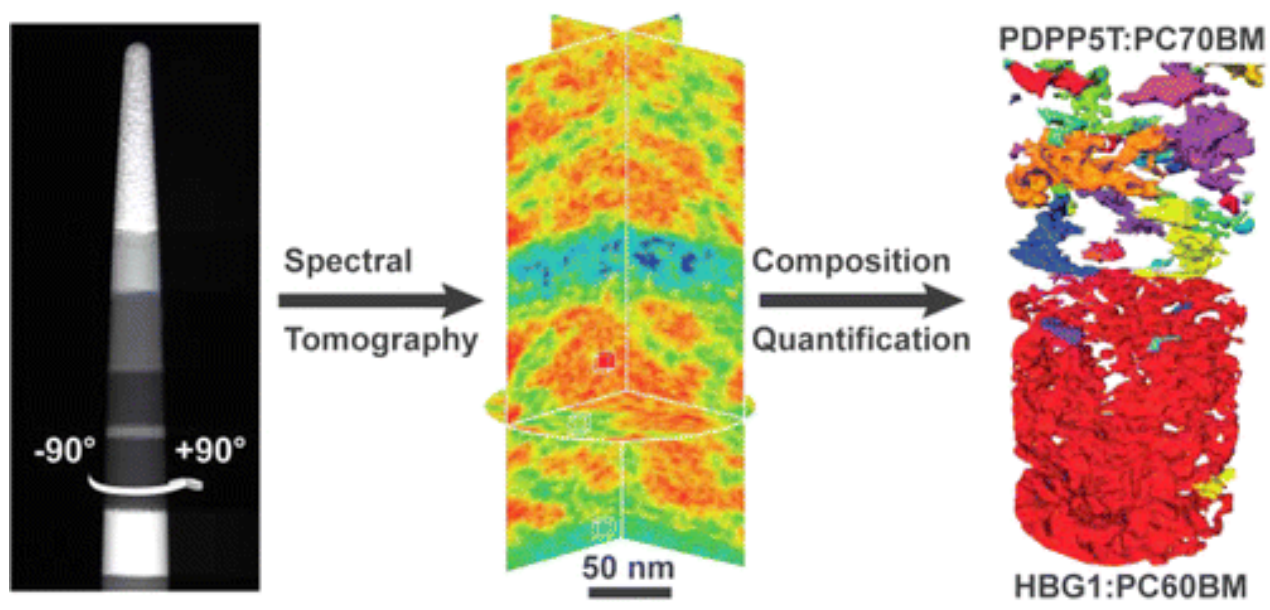

Figure 2. A needle shaped sample of an organic photovoltaic tandem cell was investigated by spectral tomography. By reconstructing entire volume plasmon spectra, 3D structural correlations and compositions could be investigated. 